\title{
DESCOMPASSO ENTRE ESTILO DE ENSINO/APRENDIZAGEM E OS OBJETIVOS DOS ALUNOS
}

\author{
MURAD, Carla Regina Rachid Otavio ${ }^{1}$
}

Recebido em: 2009.12.12

Aprovado em: 2010.02.22

ISSUE DOI: $10.3738 / 1982.2278-360$

RESUMO: Existem dois estilos cognitivos básicos de aprendizagem e de ensino que são compostos por cinco dimensões: sensorial/intuitivo; visual/global; ativo/reflexivo; seqüencial/global e dedutivo/indutivo. O foco desta pesquisa recairá sobre esta última dicotomia, que se refere às duas formas de se processar informação. Trata-se de um estudo de caso com 17 sujeitos e uma professora-pesquisadora em que verificou-se um descompasso entre as preferências da professora (priorizar o uso da língua) e os objetivos dos alunos (aprender gramática). Para contornar as dificuldades encontradas devido a esse descompasso, concluiu-se que o conteúdo de um curso de línguas deve ser trabalhado dedutiva e indutivamente, a fim de atender aos diferentes estilos de aprendizagem. Além disso, os objetivos (ou interesses) do grupo devem ser priorizados, sempre que possível, sobretudo no caso de aprendizes adultos.

Palavras-chave: Estilos cognitivos. Língua inglesa. Aprendizagem de línguas.

\section{A MISMATCH BETWEEN TEACHING AND LEARNING STYLES AND THE STUDENTS'AIMS}

SUMMARY: There are two basic learning and teaching cognitive styles which are subdivided in five dimensions: sensorial/intuitive; visual/global; active/reflexive; seqüencial/global and deductive/inductive. The focus of this research will be on this last dicothomy which refers to two ways of information processing. This is a case study with seventeen subjects and one teacher-researcher in which a mismatch between teacher's preferences (to emphasize language use) and students'objectives (learning grammar) was verified. In order to minimize the dificulties found due to this mismatch one can conclude that the content of a language course should be taught deductive and inductively in order to encompass different learning styles. Moreover, the objectives (or interests) of the group must be priorized as much as possible, specially when it comes to adult learners.

Keywords: Congnitive styles. English language learning. Applied linguistics.

\section{INTRODUÇÃO}

Este artigo, que se constitui um recorte de pesquisa de mestrado em Linguística Aplicada, especificamente na área de ensino e aprendizagem de inglês como língua estrangeira (LE), tem como objetivo priorizar o estudo dos estilos cognitivos, a fim de tentar equacionar um impasse verificado em sala de aula de inglês como LE. Para tal, fundamentouse o trabalho no arcabouço teórico da Linguística e da Psicologia Educacional do ponto de vista da interação entre professor-aluno em sala de aula de L.E.

\footnotetext{
${ }^{1}$ Professora Mestre em linguística aplicada ao ensino e aprendizagem de inglês como língua estrangeira.e-mail: carlamurad@gmail.com
} 


\section{PRESSUPOSTOS TEÓRICOS}

\section{Estilos de ensino e de aprendizagem}

Cada indivíduo tem uma forma predominante de processar a informação - indutiva ou dedutivamente. A ênfase está em "como" o indivíduo processa informação e não "o que" ou “quanto" é processado. Segundo Witkin et al.. (1977), “cognitive styles are concerned with the form rather than content of cognitive activity. They refer to individual differences in how we perceive, think, solve problems, learn, relate to others, etc. The definition of cognitive styles is thus cast in process terms". No entanto, esse conceito foi ampliado em 1982, a partir de termos mais abrangentes "it is a pervasive dimension of individual functioning, showing itself in the perceptual, intellectual, personality and social domains, and connected in its formation with the development of the organism as a whole”.

Os estilos de aprendizagem foram extensivamente discutidos na literatura sobre psicologia educacional e no contexto de aprendizagem de línguas (OXFORD, 1990; OXFORD et al., 1991; WALLACE; OXFORD, 1992; OXFORD; EHRMAN, 1993). Ao investigar o assunto, observa-se que existem vários termos, sendo que mais de 30 instrumentos de avaliação de estilo de aprendizagem já foram desenvolvidos nos últimos trinta anos. Em seu artigo sobre o tema, Jones (1998,) cita os seguintes como relevantes para a área de línguas: "Field dependence/independence", cunhados por Witking; Goodenough (1981), "holist/serialist" (PASK, 1988), "Impulsive/Reflective" (KAGAN et al., 1964), "cognitive complexity/ simplicity" (BIERI, 1961), "tolerance for unrealistic experiences" (KLEIN et al, 1962).

Alguns estudiosos os consideram estáticos por serem características construídas internamente nos indivíduos. Witking ; Goodenough já falam que os estilos são "stable over time”, isto é, que são traços estáveis que permanecem consistentes por muitos anos. Porém, afirmam que não são necessariamente de origem genética, dizendo, "that does not imply that they are unchangeable" e que, portanto, podem mudar. Brown (1980) sugere que os indivíduos apresentam tendências entre um estilo e outro mas que diferentes contextos evocam diferentes estilos em um indivíduo. Ele levanta a possibilidade de que "perhaps an intelligent and successful person is one who is bicognitive-one who can manipulate both ends of a cognitive style continuum”.

Esse argumento torna-se significativo quando pensamos na aprendizagem de línguas como um processo. Kolb (1984) concebe a aprendizagem "as a continuum process grounded in experience" e descreve os estilos de aprendizagem da seguinte maneira: "Learning styles are conceived not as fixed personality traits but as possibility-processing structures resultem from unique individual programming of the basic but flexible structure of human learning. 
These possibility-processing structures are best thought of as adaptive states or orientations that achieve stability through consistent patterns of transaction with the world." Acreditamos que o processo de aprendizagem, descrito por Kolb, pode se assemelhar ao processo de aprendizagem de uma língua estrangeira. As inúmeras possibilidades de métodos, técnicas, atividades, tornam o contexto ensino/aprendizagem de línguas estrangeiras um campo a ser explorado, tanto pelo aluno quanto pelo professor. Por se tratar de um contexto específico, cheio de novidades e de possibilidades, os indivíduos ainda estão testando suas hipóteses, estudando os riscos, delimitando a extensão; enfim, tentando entender a vasta gama de variáveis que incidem nesse processo. Nesse sentido, podemos dizer que o estilo, tanto de ensino quanto de aprendizagem, permanece um longo tempo em formação.

É nesse sentido que optamos por utilizar o esquema multidimensional de Felder e Silverman (1988) neste trabalho. Felder ; Silverman propõem um esquema composto de 5 dimensões dicotômicas que classifica os indivíduos de acordo com uma escala, relativa aos modos como recebem e processam informações. As dimensões propostas, por Felder, estão baseadas na teoria sobre Tipos Psicológicos de Jung. Jung (1971) concebe a sensação e a intuição como as duas formas através das quais as pessoas tendem a perceber o mundo. A sensação envolve a observação e a reunião de dados, através dos sentidos. A intuição envolve a percepção direta via subconsciente, isto é, acessando a memória (especulando e imaginando).

Felder ; Henriques alertam que é comum, nos estágios iniciais de aprendizagem de línguas, haver uma ênfase maior na repetição ou drills e memorização de vocabulário e gramática, atividades típicas no ensino fundamental e médio, no Brasil, tornando o aprendizado mais interessante para os sensoriais do que para os intuitivos. Por outro lado, professores intuitivos podem passar rápido demais pelo vocabulário básico e regras gramaticais, devido à sua vontade de chegar ao material mais interessante da aula, ou seja, a complexidades gramaticais, nuances de tradução, conceitos linguísticos e considerações culturais. A ênfase demasiada nesses tipos de tópico, apesar de interessante para o intuitivo, poderá prejudicar a construção de blocos básicos de linguagem, necessários nesse estágio, prejudicando os sensoriais que podem começar a ficar para trás, parando de executar as tarefas e, consequentemente, fracassando nas provas.

\section{Estilos de ensino e conflitos em sala de aula: implicações para a interação}

Como vários estudos sobre estilo cognitivo indicam (WITKIN; GOODENOUGH, 1982), os professores são igualmente influenciados e guiados pelos seus estilos na hora de ensinar. Assim como os alunos que aprendem de diversas maneiras--vendo e ouvindo, 
refletindo e agindo, raciocinando lógica e intuitivamente, memorizando e visualizando-os professores também ensinam de modos variados. Alguns lêem, outros demonstram ou discutem, alguns enfatizam a memorização e outros, a compreensão. Ambos, alunos e professores tendem a operar suas atividades de ensinar e aprender segundo seus estilos de ensino e aprendizagem, que sempre podem divergir e/ou convergir em sala de aula.

Professores mais indutivos favorecem situações que permitem uma maior interação com os alunos, ao passo que os dedutivos preferem situações de ensino mais impessoais e se orientam pelo aspecto mais cognitivo, ou intelectual do ensino.

Os componentes dos estilos de ensino e aprendizagem são paralelos entre si. Por exemplo, um aluno de percepção sensorial, provavelmente apresentará um melhor aproveitamento com um professor que enfatize fatos, ao invés de conceitos abstratos. De um modo análogo, um aluno de percepção visual se sentirá mais à vontade com um professor que utilize vídeos, fotos, figuras.

Na área de línguas, o estudo de Pask (1976) sobre os estilos holista/serialista reforça essa evidência. Quando o estilo de ensinar está em sintonia com o estilo de aprender, "the student will learn more quickly and retain the information for longer. Conversely a mismatched condition leads to grossly inferior performance”. Witkin et al. (1977) reforçam essa teoria, afirmando que, quando os estilos estão em sintonia, há uma maior chance de ocorrer atração interpessoal, o que ao nosso ver, favorece a interação.

$\mathrm{Na}$ área de ensino/aprendizagem de segunda língua, Heinrich (1993), que analisou a interação entre uma professora de português para dois americanos de estilos opostos, constatou que, devido aos seus estilos, os alunos privilegiavam a dedução ou a indução durante as aulas. Ao centrar seu ensino nos alunos, deixou o método em segundo plano e entrou em sintonia com os estilos de aprendizagem de seus alunos, facilitando, assim, o processo de aprendizagem de ambos.

Sintonizar estilo de ensino com estilo de aprendizagem é uma forma de superar o que Oxford; Lavine (1992) chamam de "style wars in the languagem classroom”. Elas afirmam que "style conflicts will not arise in the first place and [...] learning my occur far more efficiently". No entanto, questões pragmáticas e filosóficas têm adicionado maior complexidade ao tema. Reid (1987) argumenta que os alunos precisam desenvolver técnicas de compensação, necessárias para situações de conflito em potencial, como por exemplo, quando se acham expostos a uma variedade de métodos de ensino e tarefas acadêmicas. As próprias pesquisadoras reconhecem que a flexibilidade é uma habilidade importante para a vida profissional "in which people are put into jobs regardless of style and in which one is expected to deal closely with a variety of people”. Witkin et al. (1977) advertem que, para o 
educador, é importante desenvolver flexibilidade e versatilidade no comportamento dos indivíduos, como importantes metas educacionais: "for the educator, the development of greater diversity in behaviors within individuals seems as important na objective as the recognition and the utilization of diversity among individuals."

A mobilidade entre estilos de aprendizagem é particularmente importante no contexto da abordagem comunicativa, pois pode beneficiar alunos e professores.

No que se refere ao professor, o mais prático e razoável é sensibilizá-lo quanto às possíveis implicações que o seu estilo de ensino poderá ter e em função dos estilos de seus alunos, para que ele possa diversificar sua forma de ensinar. Segundo Bertini (apud JONES, 1998), "[t]he sensitizing of a teacher, and perhaps the student, to the complexity of reciprocal interaction, and equipping them with some specific tools for handling those interactions indeed seems an important step toward achieving the goal of increased teacher effectiveness".

O objetivo da abordagem centrada nos estilos de ensino/aprendizagem é equilibrar as diferenças cognitivas entre os aprendizes. Exatamente por priorizar a cognição, com variáveis previsíveis, é mais fácil verificar porque alguns aprendizes têm melhor desempenho que outros. A partir de uma base concreta (a organização do material), fica mais fácil, para o professor, diminuir a barreira entre o conteúdo da disciplina e a forma de organizá-lo. Principalmente no estruturalismo, o método desempenhava o papel central em aprendizagem de línguas (Método Tradicional, Método Direto e Método Áudio-lingual). A partir da abordagem comunicativa, tem-se privilegiado os atores-professores e alunos-de formas variadas. Graças a inúmeros pesquisadores, entre eles Prabhu (1990) e Wrigley (1993), o lugar reservado aos métodos tem sido questionado. A questão de Prabhu ("Melhor para quem?") chama a atenção para um fato importante: as diferenças entre o ponto de vista dos professores e alunos não têm sido levadas em conta nessas discussões.

A utilização exclusiva de um método, atividade ou abordagem em específico, desfavorece algumas pessoas e privilegia outras em sala de aula, ocasionando uma falta de sintonia, evidenciada na interação professor-aluno em sala de aula. Oxford ; Lavine (1992) mostram que o descompasso entre estilos de ensino e aprendizagem pode ser conflitante a ponto de se tornar uma verdadeira "batalha" ou "guerra" em sala de aula e relatam duas situações. A primeira, em que uma professora "Global" (indutiva) despertava nos alunos (dedutivos) uma reação desfavorável ao privilegiar, em excesso, atividades comunicativas; e a segunda, na qual um professor "Analítico" (dedutivo) favorecia alunos do mesmo estilo, gerando aversão naqueles cujo estilo tinha orientação oposta. 
De um lado, os alunos criticam os professores e atribuem-lhes a culpa pela falta de interesse ou motivação, pelas notas baixas e até desistências ("drop-outs"). De outro, os professores se sentem tão confrontados e desafiados pelos "desvios de comportamento" dos alunos que até começam a questionar sua própria competência como professores. Como se vê, buscava-se e ainda se busca um culpado: o método, o professor ou aluno.

A partir da ótica das diferenças individuais, se colocarmos, num tribunal, esses três "réus", veremos que todos podem se isentar da culpa e até começar a se beneficiarem dela. No nosso ponto de vista, o que ocorre é um descompasso, uma falta de sintonia, vivida por aqueles cujos estilos de ensino e aprendizagem têm uma orientação (extremamente) oposta. A hipótese aqui compartilhada com Felder; Henriques (1995) é a de que, na grande maioria das aulas de línguas, "as necessidades dos alunos, com fortes preferências ou tendências de aprendizagem, não são atendidas pelas abordagens tradicionais de ensino."

Uma das propostas para que a interação professor-aluno não se torne um campo de batalhas perdidas é integrar os pontos de vista dos alunos e professores através de uma abordagem centrada nos estilos. Para tal, acreditamos ser essencial uma postura de desvelo por parte dos sujeitos e principalmente pelo professor que é o líder da interação em sala de aula. Sua atitude deverá ser condizente com as reais necessidades de seus aprendizes. Para verificar isso, ele deverá, primeiramente, procurar fazer uma reflexão sobre sua própria prática pedagógica, inclusive para poder, se for o caso, introduzir mudanças nesse cenário. É somente através do autoconhecimento que o professor poderá entender melhor o seu estilo individual e os de seus alunos para, com isso, trabalhar no sentido de um melhor desempenho dos mesmos.

Assim como os alunos têm preferências ou estilos de aprendizagem próprios, ao organizar suas aulas, os professores também têm. Por isso, estes organizam suas aulas, conscientemente ou não, tomando como base as suas próprias preferências. Com isso, acabam priorizando um tipo de saber em detrimento do outro. Um professor que tende à indução, por exemplo, usa atividades que privilegiam o processo de aquisição em sala de aula, ao passo que um professor dedutivo planeja suas aulas de modo a favorecer a aprendizagem de gramática.

Do ponto de vista educacional, se o foco nos métodos tem excluído uma parte dos alunos, o foco nos estilos pode ser uma tentativa no sentido de equilibrar as diferenças, pois busca contemplar as necessidades de todos os alunos, sem distinção. Segundo Brown (1980), "styles can contribute significantly to the construction of a unified theory of second language acquisition”. Se a aquisição pode ocorrer na sala de aula de línguas, o foco nos indivíduos, por incluir variáveis mais abrangentes, oferece uma nova perspectiva aos estudos sobre o 
processo ensino/aprendizagem não só de segunda língua (L2) mas também de língua estrangeira (LE). A pesquisa centrada nos estilos de ensino e de aprendizagem aponta para um fator, que, ao nosso ver, é mais fácil de ser observado e controlado em sala de aula, pois tem a ver com a cognição. A consciência de que as variáveis de estilo podem influenciar tanto positiva quanto negativamente o processo de ensino/aprendizagem de línguas poderá trazer uma melhor compreensão dos fatores que incidem sobre o processo de aquisição/aprendizagem e ensino de línguas, ao invés de se procurar um culpado.

\section{METODOLOGIA DA PESQUISA}

Os dados foram levantados durante um semestre na disciplina de gramática da língua inglesa em um curso de tradução oferecido por uma instituição de ensino superior particular paulista, com 25 alunos participantes entre 18 e 45 anos. Quanto à competência linguística dos, o grupo era heterogêneo. Apesar de terem sido aprovados no vestibular, através de prova escrita e oral, os próprios SS comentavam sobre a disparidade da fluência oral deles. Alguns declararam possuir certificados de proficiência em inglês, ao passo que outros diziam não receber instrução formal há anos. No entanto, o nível deles, medido através de um teste retirado do livro How English Works (SWAN; WALTER, 1997), parecia ser equivalente, com variação de acerto entre $65 \%$ e $90 \%$.

Em seguida foi aplicado um questionário (FELDER; SILVERMAN, 1988) com o intuito de verificar os estilos (sensorial ou intuitivo) de aprendizagem dos sujeitos (SS) e o de ensino da professora-pesquisadora (PP). O questionário contém cinco dimensões, das quais dedução e indução foram posteriormente destacadas. Assim, foi possível constatar a preferência indutiva ou dedutiva dos SS e da PP. Para a PP, no entanto, foi passado um outro questionário de 126 perguntas, Myers-Briggs Type Inventory (MBTI) a fim de delinear, com mais precisão, o seu tipo psicológico. Um terceiro questionário consiste de perguntas exploratórias sobre as suas preferências com relação a uma lista de atividades didáticas (conversação, compreensão de texto, compreensão oral, etc.). O objetivo foi verificar a concepção de linguagem a fim de, posteriormente, cruzar esses resultados com os do questionário de Felder et al. (1988).

Foi gravada uma aula em vídeo, organizada segundo a preferência de (PP). O objetivo foi analisar suas decisões, escolhas de atividades e a própria organização da aula. Em seguida, com o intuito de triangularmos os instrumentos, fizemos, para os (SS), um "playback" da aula gravada. Os comentários, tecidos durante a reprodução da fita, foram gravados em áudio e 
contrastados analiticamente. Foi solicitado ainda que os alunos tomassem nota de alguns pontos específicos que quisessem, talvez, ressaltar e que fossem contribuir para a análise.

\section{DISCUSSÃO}

O objetivo principal deste trabalho foi verificar o papel dos estilos de ensino e aprendizagem em sala de aula de inglês como língua estrangeira, partindo da análise de um curso de gramática para futuros tradutores. Nesse caso específico, o objetivo dos alunos (aprender gramática) entrou em conflito com sua preferência pela organização indutiva, o que se verificou através do questionário de Felder et al. (1988). O estilo do professor, por outro lado, não interferiu na organização da aula, porque organizou o material de ambas as formas: dedutiva e indutivamente, para atingir os dois grupos de aprendizes.

A concepção de linguagem dos SS, evidenciada através de um questionário exploratório, com perguntas abertas, revelou uma orientação estruturalista, a mesma que orientava o objetivo geral do curso, visando à aprendizagem da gramática. Durante o curso, os (SS) pediam, cada vez mais, exercícios de preencher lacunas. Isso mostrava que a vontade consciente de aprender gramática era mais forte do que aprender a usar a gramática, através da comunicação, que era o que eles "gostavam" de fazer, conforme ficou constatado a partir das respostas ao questionário exploratório.

Os objetivos da escola e dos alunos batiam de frente com a concepção de linguagem da professora, que acreditava ser possível ensina gramática através do uso da língua, ou seja, que ela fosse introjetada em vez de aprendida. No entanto, ela tentava contornar a situação, dosando bem a organização do material, ou seja, procurando apresentá-lo sempre de duas formas (dedutiva e indutiva). No entanto, no decorrer do semestre, foi ficando cada vez mais evidente que os alunos queriam regras objetivas para decorarem e passarem nas provas.

Pelo fato de serem adultos com vistas a ingressarem na vida profissional a curto prazo, os alunos não abriam mão de seus pressupostos, que eram, de fato, mais fortes que seu estilo de aprendizagem. Além disso, conforme mostrou o questionário exploratório, a concepção de linguagem dos SS era fortemente estruturalista. A professora, ao apresentar o conteúdo indutivamente, percebia que, apesar de gostarem e de se saírem bem em atividades indutivas, eles resistiam porque queriam regras. Isso gerou frustração em ambas as partes. $\mathrm{Na}$ professora, que achava que todos os seus esforços estavam sendo em vão; nos alunos, que no final do semestre pediam, cada vez mais, atividades estruturadas e controladas, demonstrando uma certa falta de paciência com o processo de aprendizagem pelo qual estavam passando. 
O questionário de estilos, proposto por Felder, possibilitou toda essa reflexão sobre a prática pedagógica naquele contexto. Possibilitou à $\mathrm{PP}$ autoconhecimento e também a compreensão das variáveis que estão em jogo em sala de aula de língua estrangeira, para que elas possam ser trabalhadas de forma construtiva. Assim, tanto o ensino quanto a aprendizagem passam a fazer sentido, apesar dos descompassos.

A análise evidenciou alguns pontos essenciais, que iremos destacar, resumidamente, a seguir. Primeiro, os alunos formam um grupo heterogêneo, no que se refere (1) às formas preferenciais de aprendizagem (o que "gostam"); (2) ao estilo de aprendizagem (que reflete a forma como processam o conteúdo, ou seja, seu estilo de aprendizagem); (3) à concepção de linguagem, herdada através de cursos e professores anteriores. O/a professor/a, por sua vez,tem seu estilo próprio de aprendizagem e de ensino (que nem sempre coincidem entre si), além de também ter sido influenciada por pessoas (professores, pesquisadores, etc.) que exerceram forte influência sobre sua formação. Em meio a isso, surge a escola, que também tem suas posturas metodológicas e que segue orientações específicas.

No momento em que esses três grupos (com seus interesses e objetivos próprios) entram em contato, começam as revelações, de ambas as partes e criam-se (ou não) alguns conflitos em função das diferenças. A pergunta é: como equacionar tudo isso? A resposta a que chegamos, através deste trabalho, foi que é necessário que se tenha consciência (1) dos objetivos a serem atingidos; (2) dos estilos de aprendizagem dos sujeitos e da professora; das suas preferências por um ou por outro tipo de atividades (dedutivas ou indutivas); e (4) da concepção de linguagem (do/a professor/a e dos aprendizes), a fim de que possam entender tanto as suas próprias escolhas como a organização da aula.

Isso torna o processo de aprendizagem mais consciente, de ambas as partes, do ponto de vista cognitivo. Existem, no entanto, muitas outras variáveis, ligadas às diferenças individuais e à própria interação que se dá entre professor/a e aprendizes, que não foram contempladas neste trabalho e que precisam de uma vida para serem equacionadas.

\section{CONSIDERAÇÕES FINAIS}

Esse estudo verificou um descompasso entre as preferências da professora (priorizar o uso da língua) e os objetivos dos alunos (aprender gramática). Por serem adultos, eles têm muita clareza sobre seus objetivos. Por pretenderem ser tradutores, objetivam preencher as lacunas existentes em seu conhecimento da língua inglesa, através da prática e do estudo da gramática. Do seu ponto de vista, isso os tornaria mais confiantes e melhor preparados, como 
profissionais. Subjacente a tal crença existe uma concepção de linguagem estruturalista, que predomina nessa escola (e em muitos institutos de inglês no Brasil).

Para contornar as dificuldades encontradas, devido a esse descompasso, chegamos à conclusão de que o conteúdo de um curso de línguas deve ser trabalhado dedutiva e indutivamente, a fim de atender aos diferentes estilos de aprendizagem. Além disso, os objetivos (ou interesses) do grupo devem ser priorizados, sempre que possível, sobretudo no caso de aprendizes adultos.

\section{REFERÊNCIAS}

BIERI, J. Complexity-simplicity as a personality variable in cognitive and preferential Behavior. Functions of Varied Experience ed. D. W. Fiske ; S.R. Maddi: Homewood, 1961.

BROWN, D. Principles of language learning and teaching. Englewood Cliffs, NJ: Prentice Hall, 1980.

FELDER, R. M.; SILVERMAN, L. K. Learning styles and teaching styles. Engineering Education. Apr., 1988, p.674-681.

; HENRIQUES, E.R. Learning and teaching styles and second language

education. Foreign Language Annals, v.28, n.1, 1995, p.21-31.

HEINRICH, S.H. O papel dos estilos de ensino e de aprendizagem na interação professor/aluno. 1993. Dissertação (Mestrado), Universidade de Campinas.

JONES, S. Learning styles and learning strategies: towards learner independence. Forum for Modern Language Studies, v.34, n.2, p.115-129, 1998.

JUNG, C.G. Psychological types. Princeton, NJ: Princeton University Press, 1971.

KAGAN, J. Information process in the child: significance of analytic and reflective Attitudes. Psychological Monographs, 78, I, 1964.

KLEIN, G.C.; GARDNER, R.W.; SCHLESINGER, H.J. Tolerance for unrealistic experiences: a study of the generality of cognitive controls. British Journal of Psychology. v. 53, p.41-55, 1962 .

KOLB, D. Experiential learning: experience as the source of learning and development. Englewood Cliffs, NJ: Prentice Hall, 1984.

MYERS, I.; BRIGGS, K. The myers-briggs type indicator. Princeton NJ: Princeton University Press, 1967.

OXFORD, R.L.; EHRMAN, M.E. Second language research on individual differences. Annual Review of Applied Linguistics. v.13, p.188-205, 1993. 
OXFORD, R.; EHRMAN, M.E.; LAVINE, R.Z. Style wars: teacher-student style conflicts in the language classroom. In: MAGNANA, S. (ed.). Challenges in the 1990's for college foreign language programs. Boston: Heinle and Heinle, 1992.

OXFORD, R.L. Missing links: evidence from research on language learning styles and Strategies. In: GEORGETOWN University Round Table on Languages and Linguistics, Washington, DC: Georgetown University, 1990.

PASK, G. Styles and strategies of learning. British Journal of Educational Psychology, v.46, p.128-148, 1976.

PRABHU, N.S. There is no best method - Why? TESOL Quaterly. v. 24, n.2, p.161-176, 1990.

REID, J.M. The learning style preferences of ESL students. TESOL Quarterly. v.21, n.1, p.87-111, 1987.

SWAN, M.; WALTER, C. How english works. Oxford: University Press, 1997.

WALLACE, B.; OXFORD, R.L. Disparity in learning styles and teaching styles in the esl classroom. does this mean war? AMTESOL Journal. v.1, p.45-68, 1992.

WITKIN, C. A.et al.. Field-dependent and field-independent cognitive styles and their educational implications. Review of educational research. v.47, p.7-64, 1977.

; GOODENOUGH. Cognitive styles: essence and origins. In: FIELD

dependence and field independence: psychological issues. New York, 1981.( Monograph, 51).

WRIGLEY, H.S. One size does not fil all: educational perspectives and program practices in the US. TESOL Quaterly, v. 27, n.3, p. 449-465. 1993. 
